\title{
Classification of Possible Finite-Time Singularities by Functional Renormalization
}

\author{
S. Gluzman ${ }^{1}$ and D. Sornette ${ }^{1,2,3}$ \\ ${ }^{1}$ Institute of Geophysics and Planetary Physics \\ University of California Los Angeles, Los Angeles, CA 90095-1567 \\ ${ }^{2}$ Department of Earth and Space Sciences, UCLA \\ ${ }^{3}$ Laboratoire de Physique de la Matière Condensée \\ CNRS UMR 6622 and Université de Nice-Sophia Antipolis \\ 06108 Nice Cedex 2, France
}

October 25,2018

\begin{abstract}
Starting from a representation of the early time evolution of a dynamical system in terms of the polynomial expression of some observable $\phi(t)$ as a function of the time variable in some interval $0 \leq t \leq T$, we investigate how to extrapolate/forecast in some optimal stability sense the future evolution of $\phi(t)$ for time $t>T$. Using the functional renormalization of Yukalov and Gluzman, we offer a general classification of the possible regimes that can be defined based on the sole knowledge of the coefficients of a second-order polynomial representation of the dynamics. In particular, we investigate the conditions for the occurence of finite-time singularities from the structure of the time series, and quantify the critical time and the functional nature of the singularity when present. We also describe the regimes when a smooth extremum replaces the singularity and determine its position and amplitude. This extends previous works by (1) quantifying the stability of the functional renormalization method more accurately, (2) introducing new global constraints in terms of moments and (3) going beyond the "mean-field" approximation.
\end{abstract}




\section{Introduction}

Finite-time singularities in the dynamical equations used to describe natural systems are not always pathologies that should be thrown away or ignored but may often betray important useful information on the characteristic properties of the real system. Actually, spontaneous singularities in ordinary and partial differential equations are quite common and have been found in many well-established models of natural systems, either at special points in space such as in the Euler equations of inviscid fluids [1], 勾, in the surface curvature on the free surface of a conducting fluid in an electric field [3], in vortex collapse of systems of point vortices [4], in the equations of General Relativity coupled to a mass field leading to the formation of black holes [5], in models of micro-organisms aggregating to form fruiting bodies [6], or in the more prosaic rotating coin (Euler's disk) [7, 8]. Some more complex examples are models of rupture and material failure [9, 10, 11], earthquakes [12, 13] and stock market crashes [14, 15].

In a recent work [16], we have developed theoretical formulas for the prediction of the singular time of systems which are a priori known to exhibit a critical behavior, based solely on the knowledge of the early time evolution of an observable. From the parameterization of such early time evolution in terms of a low-order polynomial of the time variable, the functional renormalization approach introduced by Yukalov and Gluzman allows one to transform this polynomial into a function which is asymptotically a power law. The value of the critical time $t_{c}$, conditioned on the assumption that $t_{c}$ exists, can then be determined from the knowledge of the coefficients of the polynomials. Ref. [16] has tested with success this prediction scheme on a specific example and showed that this approach gives more precise and reliable predictions than through the use of the exact real power law model. This is a rather surprising and paradoxical observation in contradiction with common wisdom according to which the best and most reliable prediction should be obtained from the use of the exact underlying model of the dynamical behavior [17]. The reason why this is not always true is that our method has shown that approximate solutions can be more stable and more reliable when devised so as to maximize the criterion of stability with respect to perturbations in the functional space [16].

Here, we extend this work by offering a general classification of the possible regimes that can be defined based on the sole knowledge of the coefficients of the polynomial expansion of some observable as a function of the time variable. We mostly restrict our analysis to second-order polynomials. As a consequence, the classification can be organized in a unique way in terms of the single signed Froude parameter, ratio of the square of the velocity to the acceleration.

Let us assume that the dynamical behavior of a system is sampled to obtain a time series $\phi(t)$ in the interval $0 \leq t \leq T$. The question we address in this work is how to extrapolate/forecast in some optimal sense the future evolution of $\phi(t)$ for time $t>T$. Beyond a simple extrapolation, we ask whether it is possible to detect the germs of a finite-time singularity from the structure of the time series, and quantify the critical time and the functional nature of the singularity when present. We also aim at classifying the regimes when a smooth extremum replaces the singularity and at determining its position and amplitude.

We shall work in the framework of the functional renormalization method, which constructs the extrapolation for $t>T$ from a resummation of the time series represented by a simple model-free polynomial expansion in powers of time $t$, where $t$ is counted since the beginning of the recorded time series.

\section{Summary of the functional renormalization approach to extrapolation of times series}

The mathematical foundation of the functional renormalization approach used here can be found in earlier publications [18]-[30], which we summarize briefly. Let perturbation theory (or some fitting procedure) give for the time series $\phi(t)$ the succession of non-random approximations $\phi_{n}(t)$ where $n=0,1,2, \ldots$ enumerates the order of the increasingly 
precise approximations. The case of a polynomial expansion will be discussed below,

$$
\phi_{n}(t)=\sum_{k=0}^{n} a_{k} t^{k}, \quad n=0,1,2,3, \ldots
$$

This expansion (1) is in principle defined for $t$ sufficiently small and based on information about the time series up to the time $T$. The expansion may have no meaning if continued straightforwardly to the region of finite $t>T$. The problem of reconstructing the value of the function in some distant moment of time from the knowledge of its asymptotic expansion as $t \rightarrow 0$ is called a renormalization or resummation problem in theoretical physics. An analytical tool for the solution of this problem, called algebraic self-similar renormalization, has been developed recently [18]-[30] of which we summarize the salient points useful for the present work.

It is convenient to remove the constant term and consider the time series $p(t) \equiv \phi(t)-\phi_{0}$ represented by the sequence of polynomial approximations $p_{i}(t), i=1,2, \ldots, n$,

$$
p_{1}(t)=a_{1} t, \quad p_{2}(t)=p_{1}(t)+a_{2} t^{2}, \ldots, \quad p_{n}(t)=p_{n-1}(t)+a_{n} t^{n} .
$$

The algebraic self-similar renormalization starts by applying to the approximations (2) a simple algebraic multiplication, thus defining a new sequence, $P_{i}(t, s)=t^{s} p_{i}(t), i=1,2, \ldots, n$, with $s \geq 0$. This transformation increases the powers of the series (11) and (2). This formal manipulation effectively increase the order of the expansion and provides a trick to effectively take into consideration more points from the system trajectory. In the first part of the paper, we use the strongest form of this transformation corresponding to formally take the limit $s \rightarrow \infty$. This will be shown to provide a representation of the renormalized function as an imbedded set of exponentials associated with a singularity $\phi \sim\left(t_{c}-t\right)^{z}$, with critical exponent $z$ imposed to have an absolute value equal to 1 . This limit can thus be considered as analogous to a "mean field" regime with the mean field value of the critical exponent $z=-1$. This can for instance represent the exponent of the derivative of a quantity exhibiting a weak logarithmic divergence. In the second part of the paper, we shall relax this limit and determine both $t_{c}$ and the index $z$ self-consistently.

The second step consists in considering the sequence of transformed approximations $P_{i}(t, s)$, as a dynamical system in the discrete "order time" equal to the order $i=0,1, \ldots, n-1$ of the approximation. In order to keep the information on the system evolution with the real time $t$, it is convenient to introduce a new variable $\varphi$ and define the so-called expansion function $t(\varphi, s)$ from the equation $P_{1}(t, s)=a_{1} t^{1+s}=\varphi$, which gives $t(\varphi, s)=\left(\varphi / a_{1}\right)^{1 / 1+s}$. We then construct the discrete flow in the space of approximations indexed by the "order time" as

$$
y_{i}(\varphi, s) \equiv P_{i}(t(\varphi, s), s) .
$$

One can then write the equation of evolution in the space of approximations as a function of the discrete "order time" in the form of the functional self-similarity relation

$$
y_{i+p}(\varphi, s)=y_{i}\left(y_{p}(\varphi, s), s\right) .
$$

Expression (4) provides the necessary condition for the self-consistency of the cascade of discrete approximations and ensures the convergence of $P_{i}$ 's. Expression (4) is nothing but the application of the Caccioppoli-Banach principle for the existence of a stable fixed point (see for instance Chapter XVI of [31]).

At this stage, the efficiency of the algebraic transformation can be checked by analyzing its stability. This is done for the sequence of $P_{i}(t, s)$ 's by calculating the so-called local multipliers (essentially proportional to the exponential of Lyapunov exponents)

$$
m_{i}(t, s) \equiv\left[\frac{\partial y_{i}(\varphi \cdot s)}{\partial \varphi}\right]_{\varphi=P_{1}(t, s)} .
$$

When all $\left|m_{i}(t, s)\right|<1$, the convergence of the sequence $P_{i}$ is guaranteed. To implement concretely the calculations, we use the integral form of the self-similarity relation ( $($ )

$$
\int_{P_{i-1}}^{P_{i}^{*}} \frac{d \varphi}{v_{i}(\varphi, s)}=\tau
$$


where the cascade velocity is $v_{i}(\varphi, s)=y_{i}(\varphi, s)-y_{i-1}(\varphi, s)$ and $\tau$ is the minimal number of steps of the approximation procedure needed to reach the fixed point $P_{i}^{*}(t, s)$ of the approximation cascade. It is possible to find $P_{i}^{*}(t, s)$ explicitly and to perform an inverse algebraic transform after which the limit $s \rightarrow \infty$ is to be taken. This completes a first loop of the self-similar renormalization. This procedure can be repeated as many times as it is necessary to renormalize all polynomials which appear at the preceding steps. This is the main idea of the self-similar bootstrap [22].

Completing this program, we come to the following sequence of self-similar exponential approximants

$$
p_{j}^{*}\left(t, \tau_{1}, \tau_{2}, \ldots, \tau_{j-1}\right)=a_{1} t \exp \left(\frac{a_{2}}{a_{1}} t \tau_{1} \ldots \exp \left(\frac{a_{j}}{a_{j-1}} \tau_{j-1} t\right)\right) \ldots, \quad j=2,3 \ldots, n .
$$

and

$$
\phi_{j}^{*}\left(t, \tau_{1}, \tau_{2}, \ldots, \tau_{j-1}\right)=p_{j}^{*}\left(t, \tau_{1}, \tau_{2}, \ldots, \tau_{j-1}\right)+a_{0} .
$$

Explicitly, for the three first orders, we obtain

$$
\begin{gathered}
\phi_{2}^{*}\left(t, \tau_{1}\right)=a_{1} t \exp \left(\frac{a_{2}}{a_{1}} t \tau_{1}\right)+a_{0}, \\
\phi_{3}^{*}\left(t, \tau_{1}, \tau_{2}\right)=a_{1} t \exp \left(\frac{a_{2}}{a_{1}} t \tau_{1} \exp \left(\frac{a_{3}}{a_{2}} t \tau_{2}\right)\right)+a_{0}, \\
\phi_{4}^{*}\left(t, \tau_{1}, \tau_{2}, \tau_{3}\right)=a_{1} t \exp \left(\frac{a_{2}}{a_{1}} t \tau_{1} \exp \left(\frac{a_{3}}{a_{2}} t \tau_{2} \exp \left(\frac{a_{4}}{a_{3}} t \tau_{3}\right)\right)\right)+a_{0} .
\end{gathered}
$$

Compared to previous works [18]-[30], we introduce the following innovation to improve on the selection of the stable extrapolations (or scenarios). In order to check whether the sequence of $\phi_{j}^{*}\left(t, \tau_{1}, \tau_{2}, \ldots, \tau_{j-1}\right)$ really converges, in addition to calculating the multipliers $m_{i}(t, s)$ defined by (5), we analyze the multipliers $M_{j}\left(t, \tau_{1}, \tau_{2, . .}, \tau_{j-1}\right)$ corresponding specifically to $\phi_{j}^{*}\left(t, \tau_{1}, \tau_{2}, \ldots, \tau_{j-1}\right)$. For this, we construct again an approximation cascade as described above and define

$$
M_{j}\left(t, \tau_{1,} \tau_{2, . .}, \tau_{j-1}\right) \equiv\left[\frac{\partial p_{j}^{*}\left(\varphi, \tau_{1}, \tau_{2, . .,} \tau_{j-1}\right)}{\partial \varphi}\right]_{\varphi=P_{1}(t, 0)} .
$$

Such a definition of multipliers allows us for the first time to compare the convergence of the expansion and of the renormalized expansion, making clear the improvements that can be expected a priori from the technique. If the values of $M_{j}$ are systematically smaller than the corresponding $m_{j}$ in the region of $t \leq T$ and the overall convergence properties are improved, one can expect that the renormalized expressions will work better than the original expansion at $t>T$.

The final step consists in determining the control parameters $\tau_{1}, \tau_{2}, \ldots, \tau_{j-1}$, by expanding $\phi_{j}^{*}\left(t, \tau_{1}, \tau_{2}, \ldots, \tau_{j-1}\right)$ in the vicinity of $t=0$, and requiring that this expansion agrees term-by-term with the initial one in terms of the $\phi_{j}(t)$. For each $j$, we obtain $j-1$ self-similar approximants for the sought function (where all control parameters $\tau$ are now known functions of the parameters $a$, with $\tau_{1} \equiv 1$ ),

$$
\begin{array}{r}
\phi_{j 1}^{*}(t, 1,1 \ldots, 1), \\
\phi_{j 2}^{*}\left(t, 1, \tau_{2}, 1, \ldots, 1\right), \\
\ldots \\
\phi_{j j-1}^{*}\left(t, 1, \tau_{\left.2, \ldots, \tau_{j-1}\right)},\right.
\end{array}
$$

which differ according to the number of control functions being used. We can now construct a table of self-similar approximants, varying both $j$ and the number of controls. Accordingly, we can define the table of multipliers, varying both $j$ and the number of controls. For instance, for $j=4$, we have the following table of approximations:

$$
\phi_{21}^{*}(t)=\phi_{j}^{*}(t, 1)
$$




$$
\begin{aligned}
& \phi_{31}^{*}(t)=\phi_{3}^{*}(t, 1,1), \phi_{32}^{*}(t)=\phi_{3}^{*}\left(t, 1, \tau_{2},\right), \\
& \phi_{41}^{*}(t)=\phi_{4}^{*}(t, 1,1,1), \phi_{42}^{*}(t)=\phi_{4}^{*}\left(t, 1, \tau_{2}, 1\right), \phi_{43}^{*}(t)=\phi_{4}^{*}\left(t, 1, \tau_{2}, \tau_{3}\right) .
\end{aligned}
$$

In order to select the scenarios for the extrapolation to the future from the initial time series, one needs to examine both the properties of convergence of the sequences of multipliers $M$, and of approximants $\phi^{*}$. The best scenario is the one which exhibits the best simultaneous convergence of both sequences. The resulting limiting fixed point read from the table of approximants should then be taken for the sought extrapolation function. If there are more than one limiting points, the sought function should be constructed by taking their average with weights inversely proportional to the multipliers (see [30]). We now illustrate this general methodology on specific examples for which a finite-time singularity might exist.

\section{Classification of singular and non-singular behaviors based on second order ex- pansions}

\subsection{Definitions}

Let us now consider the simplest non-trivial case allowing for the possible existence of a finite-time singularity, namely a second-order polynomial representation

$$
\phi_{2}(t) \simeq 1+a_{1} t+a_{2} t^{2} \quad(t \rightarrow 0)
$$

of the initial time series. In the language of [32], $a_{1}$ is the "velocity" and $2 a_{2}$ is the "acceleration". The relative influence of the velocity and acceleration is quantified by the so-called Froude number [32] defined by

$$
F_{d} \equiv \frac{a_{1}^{2}}{a_{2}} .
$$

Working with the "direct" series (15), the program described in the previous section provides the sought observable, that we name $\Phi_{2}^{*}(t)$, equal to the re-summed approximant:

$$
\Phi_{2}^{*}(t)=\phi_{21}^{*}(t)=1+a_{1} t \exp \left[\frac{a_{2}}{a_{1}} t\right] .
$$

We will also study, when necessary, the inverse function $\phi_{2 I}(t)$ of $\phi_{2}(t)$,

$$
\phi_{2 I}(t)=1 / \phi_{2}(t)=1-a_{1} t+\left(-a_{2}+a_{1}^{2}\right) t^{2}+\ldots \equiv 1+b_{1} t+b_{2} t^{2}+\ldots
$$

The corresponding Froude number $F_{I}$ is

$$
F_{I} \equiv \frac{b_{1}^{2}}{b_{2}}=\frac{a_{1}^{2}}{a_{1}^{2}-a_{2}}=\frac{F_{d}}{F_{d}-1}, \quad\left(F_{d}=\frac{F_{I}}{F_{I}-1}\right) .
$$

In cases when better convergence can be achieved by working with the "inverse" series (18), the observable $\Phi_{2}^{*}(t)$ will be expressed through the approximant corresponding to the inverse series as follows:

$$
\Phi_{2}^{*}(t)=\left[\phi_{21 I}^{*}(t)\right]^{-1}=\left(1+b_{1} t \exp \left[\frac{b_{2}}{b_{1}} t\right]\right)^{-1}
$$

The convergence of our procedure is checked by estimating the value of the multiplier

$$
M_{21}(t)=\left(1+\frac{a_{2}}{a_{1}} t\right) \exp \left(\frac{a_{2}}{a_{1}} t\right) .
$$

The same expression gives the multiplier for the inversion function by changing all the coefficients $a_{i}$ 's into $b_{i}$ 's, where the $b_{i}$ 's are related to the $a_{i}$ 's via expression (18). 


\subsection{Negative velocity $a_{1}<0$ (downward trend)}

\subsubsection{Negative acceleration $a_{2}<0$}

Working with positive observables, this case corresponds to the possibility that the observable vanishes in finite time. This zero-crossing occurs at $t_{c 21}$ given by

$$
1+F_{d} Z_{d} \exp \left[Z_{d}\right]=0
$$

where $Z_{d}=\frac{a_{2}}{a_{1}} t_{c 21}$. The corresponding multiplier $M_{21}\left(t_{c 21}\right)$ is larger than 1 , signaling a possible problem with the convergence of the functional renormalization method. This may signal an instability in the time dynamics of the time series close to the zero-crossing time.

\subsubsection{Moderate positive acceleration $0<a_{2}<\frac{a_{1}^{2}}{e}$}

The observable goes again to zero in finite time. The time $t_{c 21}$ at which the observable vanishes is given again by (22). The corresponding multiplier $M_{1}\left(t_{c 21}\right)$ is now less than 1 , signaling a stable scenario.

\subsubsection{Strong positive acceleration $\frac{a_{1}^{2}}{e}<a_{2}$}

The acceleration is sufficiently positive to counter-balance the negative trend and the observable goes through a minimum before rebounding upward. The time $t_{\min }$ at which the minimum $\Phi_{\min }^{*}=1-\frac{F_{d}}{e}$ is reached is given by

$$
t_{\min }=-\frac{a_{1}}{a_{2}} .
$$

This is a very stable situation since the multiplier is zero at the minimum. Our analysis suggests that the extrapolation to the future is the most credible for this situation in the downward trend case.

\subsection{Positive velocity $a_{1}>0$ (upward trend)}

\subsubsection{Negative acceleration: $a_{2}<0$}

The observable increases up to a maximum $\Phi_{\max }^{*}=1-\frac{F_{d}}{e}$ and then decreases after it. The time $t_{\max }$ of the maximum is given by

$$
t_{\max }=-\frac{a_{1}}{a_{2}} .
$$

This is a very stable situation since the multiplier $M_{21}$ is less than 1 for arbitrary time $t$ and is zero at the maximum.

\subsubsection{Positive acceleration}

This case requires the inversion of the time series, since the multipliers of the direct series are always larger than 1: the expansion is not convergent and does not supply any information on the real-axis singularities. The inversion maps these two cases on the cases previously considered with multipliers smaller than 1 . In addition, the corresponding series has alternating signs, suggesting that finite-time singularity is indeed present.

Moderate positive acceleration: $0<a_{2}<\frac{e-1}{e} a_{1}^{2} \quad$ The observable increases up to a maximum and then decreases after it. The time $t_{\max }$ of the maximum is given by

$$
t_{\max }=-\frac{b_{1}}{b_{2}}=\frac{a_{1}}{a_{1}^{2}-a_{2}},
$$


and the value at maximum is

$$
\Phi_{\max }=\left(1-\frac{F_{I}}{e}\right)^{-1}
$$

This solution is very stable and thus very credible as the multiplier for $\phi_{21 I}^{*}(t)$ is always smaller than 1 and vanishes at the maximum. The original function is obtained by the inversion $\Phi_{2}^{*}(t)=\left(\phi_{21 I}^{*}(t)\right)^{-1}$ (see also equation (18)).

Large positive acceleration: $\frac{e-1}{e} a_{1}^{2}<a_{2}$ or $F_{d}<\frac{e}{e-1} \quad$ There is an upward finite-time singularity at $t_{c 21}$ given by the zero of the inverted renormalized expansion, i.e., by the solution of

$$
\phi_{21 I}^{*}\left(t_{c 21}\right)=1+F_{i} Z_{i} \exp \left[Z_{i}\right]=0
$$

where $Z_{i}=t_{c 21} \frac{a_{2}-a_{1}^{2}}{a_{1}}$. The multiplier for $\left.\phi_{21 I}^{*}(t)\right)$ is smaller than 1 , signaling the convergence of the theoretical procedure for the inverse functions. Recall again that, in order to return to the original function, the inversion $\Phi_{2}^{*}(t)=$ $\left(\phi_{21 I}^{*}(t)\right)^{-1}$ should be performed, leading to a divergence of the original observable at the point where its inverse crosses zero.

\subsection{Example}

Let us consider a function with known singular behavior and compare the results obtained by means of self-similar approximations with the exact values. We consider the function $1 / \cos (t)$ which was found [33] to describe the timeevolution of the crack length of a self-consistent model of damage and used [16] for prediction tests. Starting from $t=0$, the function $1 / \cos (t)$ possesses a singularity at $t=\pi / 2 \approx 1.5708$, and its expansion up to second order in powers of $t^{2}$ reads

$$
\phi_{2}(t) \simeq 1+1 / 2 t^{2}+5 / 24 t^{4}+\ldots
$$

Note that, since $1 / \cos (t)$ is an even function, the relevant variable for the expansion is indeed $t^{2}$ and our previous classification must be applied to the expansion (27) up to second order in $t^{2}$ (i.e., to fourth-order $t^{4}$ ).

The corresponding Froude number is $F_{d}=1.2$ and obeys the condition $1<F_{d}<\frac{e}{e-1}$, i.e., the previous section shows that one can anticipate a finite-time singularity, only on the basis of the quadratic polynomial expansion. The expansion of the inverse of $\phi_{2}(t)$ reads

$$
\phi_{2 I}(t) \simeq 1-1 / 2 t^{2}+1 / 24 t^{4}+\ldots
$$

As expected from our previous analysis in terms of the stability of the renormalization flow quantified by the multipliers, the position of the critical time is better estimated from the zero of $\phi_{21 I}^{*}\left(t_{c 21}\right)=0$, which gives $t_{c 21}=$ 1.56645 , compared to the value $t_{20}=1.59245$ suggested from the condition $\phi_{20 I}\left(t_{20}\right)=0$. This last value $t_{20}$ is nothing but the prediction of the critical time from the "bare" polynomial expansion. We refer to (14) for the definition of $\phi_{21 I}^{*}(t)$ and $\phi_{20 I}(t)$. This shows that using a control parameter, which ensures that the renormalized expansion retrieves the polynomial expansion, improves the prediction of the critical time.

\section{Illustration of the selection of scenarios by the convergence of higher-order ap- proximants}

We now exploit the previous example of the function $1 / \cos (t)$ described in section 3.4 to illustrate how the concept of convergence of the approximants developed in section II can be used for an improved determination of the singularity.

Higher-order expansions are given by

$$
\phi_{3 I}(t) \simeq \phi_{2 I}(t)-1 / 720 t^{6}+\ldots, \quad\left(b_{3}=-1 / 720\right)
$$


and

$$
\phi_{4 I}(t) \simeq \phi_{3 I}(t)+1 / 40320 t^{8}+\ldots, \quad\left(b_{4}=1 / 40320\right) .
$$

The corresponding higher order approximants are

$$
\Phi_{3}^{*}(t)=\left(\phi_{3 I}^{*}(t)\right)^{-1}=\left(1+b_{1} t^{2} \exp \left(\frac{b_{2}}{b_{1}} t^{2} \exp \left(\frac{b_{3}}{b_{2}} \tau_{2} t^{2}\right)\right)\right)^{-1},
$$

with

$$
\tau_{2}=1-\frac{b_{2}^{2}}{2 b_{1} b_{3}}
$$

and

$$
\Phi_{4}^{*}(t)=\left(\phi_{4 I}^{*}(t)\right)^{-1}=\left(1+b_{1} t^{2} \exp \left(\frac{b_{2}}{b_{1}} t^{2} \exp \left(\frac{b_{3}}{b_{2}} \tau_{2} t^{2} \exp \left(\frac{b_{4}}{b_{3}} \tau_{3} t^{2}\right)\right)\right)\right)^{-1}
$$

with

$$
\tau_{3}=-\frac{b_{3}}{12 b_{1} b_{2} b_{4}\left(b_{2}^{2}-2 b_{1} b_{3}\right)} \cdot\left(24 b_{1}^{2} b_{4} b_{2}+5 b_{2}^{4}-12 b_{1}^{2} b_{3}^{2}-12 b_{1} b_{3} b_{2}^{2}\right) .
$$

As explained in section II, the control parameters $\tau_{2}$ and $\tau_{3}$ are determined from the condition that the expansion of the approximants at small $t$ coincide with the perturbative expression of the initial polynomial representation.

For each approximant of a given different order and with a given number of control parameters, we obtain a prediction for the position of the singularity obtained from the condition of zero-crossing of the inverse approximant. Let us compare these values between them and with the critical time derived from the corresponding initial polynomial fit. We find the following results:

$$
\begin{aligned}
& t_{c 21}=1.56645, \\
& t_{c 31}=1.55134, \quad t_{c 32}=1.57067, \\
& t_{c 41}=1.55193, \quad t_{c 42}=1.57048, \quad t_{c 43}=1.57079 ;
\end{aligned}
$$

corresponding to the following multipliers defined by (12):

$$
\begin{aligned}
M_{21}\left(t_{c 21}\right) & =0.6484, \\
M_{31}\left(t_{c 31}\right) & =0.68955, M_{32}\left(t_{c 32}\right)=0.63708, \\
M_{41}\left(t_{c 41}\right) & =0.68743, M_{42}\left(t_{c 42}\right)=0.63772, M_{43}\left(t_{c 43}\right)=0.63663 .
\end{aligned}
$$

The values $t_{c 21}, t_{c 31}, t_{c 32}, t_{c 41}, t_{c 42}$ and $t_{c 43}$ should be compared with the values determined from the "bare" polynomials

$$
t_{20}=1.59245, \quad t_{30}=1.56991, \quad t_{40}=1.57082 .
$$

The convergence of this last sequence can be analyzed by looking at the corresponding sequence of multipliers $m_{i}(t, 0)$ defined by (5):

$$
m_{2}\left(t_{02}\right)=0.57735, m_{3}\left(t_{03}\right)=0.63985, m_{4}\left(t_{04}\right)=0.63651 .
$$

Overall these results show indeed a good convergence for $t_{c 41}, t_{c 42}$ and $t_{c 43}$ by increasing the number of control parameters and along the diagonal $t_{c 21}, t_{c 32}, t_{c 43}$ by increasing simultaneously both the order of the polynomial and the number of control parameters. The same observation holds for the multipliers $M$. In contrast, the bare polynomials give a slower non-monotonous convergence. This confirms the improvement brought by our scheme to obtain a better determination of the critical time $t_{c}$. 


\section{Non-local control through the moments of the function to predict}

Section II and our subsequent tests have shown that the control parameters provide a mean to improve the extrapolation to the future by imposing some constraint on the approximants. Up to now, we have used the constraint that the approximants must retrieve the "bare" polynomial expansions at small times $t$. This corresponds to constraints which are local in time.

It is interesting and potentially useful to investigate the possibility of using more global "non-perturbative" constraints. A possible example is when, either from a priori theoretical knowledge or from experimental or empirical measurements, we get hold of the first $j-1$ moments $\mu_{i}, i=1,2 . ., j-1$ of the sought function $\phi(t)$ in some interval $[0, T]:$

$$
\mu_{i}=\int_{0}^{T} t^{i-1} \phi(t) d t
$$

Our notation means that, for $j=2$, we know only the zero moment ("mass" or integral $\phi(t)$ from 0 to $T$ ), for $j=3$, we know both the zeroth and first moment, etc...

Endowed with this knowledge of the first $j-1$ moments, we can condition the control parameters $\tau_{1,}, \tau_{2, . .} \tau_{j-1}$ demanding that the reconstructed approximants have exactly the right values of their moments:

$$
\int_{0}^{T} \phi_{j}^{*}\left(t, \tau_{1}, \tau_{2}, \ldots, \tau_{j-1}\right) t^{i-1} d t=\mu_{i} .
$$

For $j=2$, we have one equation for $\tau_{1}$ :

$$
\int_{0}^{T} \phi_{2}^{*}\left(t, \tau_{1}\right) d t=\mu_{0}
$$

For $j=3$, we obtain two equations for $\tau_{1}$ and $\tau_{2}$ :

$$
\int_{0}^{T} \phi_{3}^{*}\left(t, \tau_{1}, \tau_{2}\right) d t=\mu_{0}, \quad \int_{0}^{T} \phi_{3}^{*}\left(t, \tau_{1}, \tau_{2},\right) t d t=\mu_{1}
$$

For $j=4$, we have three equations for $\tau_{1}, \tau_{2}$ and $\tau_{3}$ :

$$
\int_{0}^{T} \phi_{4}^{*}\left(t, \tau_{1}, \tau_{2}, \tau_{3}\right) d t=\mu_{0}, \quad \int_{0}^{T} \phi_{4}^{*}\left(t, \tau_{1}, \tau_{2}, \tau_{3}\right) t d t=\mu_{1}, \quad \int_{0}^{T} \phi_{4}^{*}\left(t, \tau_{1}, \tau_{2}, \tau_{3}\right) t^{2} d t=\mu_{2} .
$$

Based on these conditions, two different problems seem most natural. The first one is to construct an approximate representation of the function $\phi(t)$ in the same interval $[0, T]$ where moments are given or measured. In the case where the moments are obtained through some experimental procedure leading to some measurement errors, this first problem amounts to filter out the noise in the measurement interval $[0, T]$. The second problem that we shall address here consists in extrapolating to times $t>T$.

Using the previous example of the function $1 / \cos (t)$, let us consider the following case $T=\sqrt{2}$. This value is "natural" as it is the root of $1+b_{1} t^{2}$. Constraining the control parameters by the knowledge of the moments in the interval $[0, \sqrt{2}]$, we obtain the corresponding approximants. The analysis of the zero of the inverse approximants give the following estimations for the critical times and the corresponding multipliers

$$
\begin{aligned}
t_{c 21} & =1.56888, \quad t_{c 32}=1.57077, \quad t_{c 43}=1.570796, \\
M_{21}\left(t_{c 21}\right) & =0.64388, \quad M_{32}\left(t_{c 32}\right)=0.63678, \quad M_{43}\left(t_{c 43}\right)=0.63662 .
\end{aligned}
$$

Notice the extremely good quality of the convergence of both the critical times and of the multipliers.

It is also possible to use an hybrid approach, where some control parameters are obtained from the agreement with the polynomial expansion at small time $t$, while the remaining ones are determined from the conditions on the known moments. As an illustration, we show the fifth-order approximant

$$
\phi_{5 I}^{*}\left(t, \tau_{1}, \tau_{2}, \tau_{3}, \tau_{4}\right)=\left(1+b_{1} t^{2} \exp \left(\frac{b_{2}}{b_{1}} \tau_{1} t^{2} \exp \left(\frac{b_{3}}{b_{2}} \tau_{2} t^{2} \exp \left(\frac{b_{4}}{b_{3}} \tau_{3} t^{2} \exp \left(\frac{b_{5}}{b_{4}} \tau_{4} t^{2}\right)\right)\right)\right)\right),
$$


where $\tau_{1}=1$ is conditioned by the polynomial expansion and the other control parameters should be calculated from the system of equations

$$
\begin{aligned}
\int_{0}^{T} \phi_{5 I}^{*}\left(t, 1, \tau_{2}, \tau_{3}, \tau_{4}\right) d t & =\mu_{0}, \\
\int_{0}^{T} \phi_{5 I}^{*}\left(t, 1, \tau_{2}, \tau_{3}, \tau_{4}\right) t d t & =\mu_{1}, \\
\int_{0}^{T} \phi_{5 I}^{*}\left(t, 1, \tau_{2}, \tau_{3}, \tau_{4}\right) t^{2} d t & =\mu_{2} .
\end{aligned}
$$

Note that we do not even need to know the exact values $b_{3}, b_{4}$ and $b_{5}$ of the polynomial expansion since they can be included in the corresponding controls. This results from the fact that the constraints on the moments overwhelm the initial information on the coefficients of the polynomial expansion. We find $t_{c 54}=1.570796$ in extremely good agreement with the exact critical time $t_{c}=\pi / 2=1.5707963$.

\section{Classification and forecasting of critical times beyond mean-field}

We now use the formalism of section II and relax the condition $s \rightarrow+\infty$ on the exponent of the algebraic transformation, which amounted to impose the mean-field value $z=-1$ of the critical exponent. The control exponent $s$ will be determined from the optimization of the convergence and the stability of the renormalization flow. according the general principles developed by Yukalov and Gluzman [23]-[30].

\subsection{General procedure}

Consider as before an expansion of an observable $\phi(t)$ in powers of a variable $t$ (time) given by $\phi_{k}(t)=\sum_{n=0}^{k} a_{n} t^{n}$ where $a_{0}=1$ without loss of generality by suitable normalization. The method of algebraic self-similar renormalization [23]-[25] gives the following general recurrence formula for the approximant of order $k$ as a function of the expansion $\phi_{k-1}(t)$ up to order $k-1$ :

$$
\phi_{k}^{*}(t)=\phi_{k-1}(t)\left[1-\frac{k a_{k}}{s} t^{k} \phi_{k-1}^{k / s}(t)\right]^{-s / k} \equiv\left[\phi_{k-1}^{-k / s}(t)-\frac{k a_{k}}{s} t^{k}\right]^{-s / k},
$$

where, in general, $s=s_{k}(t)$ depends on the approximation number $k$ and on the variable $t$. These approximants automatically agree with their corresponding polynomial expansions and the sole way to impose some control is to restrict $s$ using some conditions of rather general nature such as convergence of the sequence of approximants.

In the sequel, we assume that only the second-order expansion is available. In order to determine the critical exponent $z$, we follow Yukalov and Gluzman [25] and construct the two approximants available from the knowledge of the two coefficients $a_{1}$ and $a_{2}$. They can be readily obtained from the general formula (39). The first order approximant is simply

$$
\Psi_{1}^{*}(t)=\left(1-\frac{a_{1}}{s_{1}} t\right)^{-s_{1}}
$$

Representing $\phi_{2}(t)$ as $\phi_{2}(t)=1+a_{1} t\left(1+\frac{a_{2}}{a_{1}} t\right)$ and applying the general formula to the expression in brackets, we obtain the second order approximant

$$
\Psi_{2}^{*}(t)=1+a_{1} t\left(1-\frac{a_{2}}{a_{1} s_{2}} t\right)^{-s_{2}}
$$

Let us assume further that $s_{1}=s_{2}=s$, where $s$ is the limiting value of the control function of the algebraic transformation at the critical point. It is apparent from the form of (39) that $s$ plays the role of the critical index $z$. As it was explained in [25], this is justified in the vicinity of a stable fixed point. 
The condition of maximum stability of the renormalization flow is equivalent to imposing that the difference $\Psi_{2}^{*}-\Psi_{1}^{*}$ be a minimum with respect to the set of parameters (the so-called minimal difference condition). We discuss below an application of the technique applied to direct second-order expansions.

In the present work, we are interested in testing for the existence of a finite-time singularity or critical point. Looking for such an occurrence, we need to solve the minimal difference condition which amounts to look for the solutions of the two equations in terms of the two variables $t_{c}$ and $s$ :

$$
\Psi_{1}^{*}\left(t_{c}, s\right)=0 \quad \text { and } \quad \Psi_{2}^{*}\left(t_{c}, s\right)=0 .
$$

The vanishing of $\Psi_{1}^{*}$ given by (40) gives

$$
t_{c}=s / a_{1}
$$

The second condition $\Psi_{2}^{*}=0$ with (41) provides an estimate $s$ for the critical index. In terms of Froude parameter $F_{d}$ defined by (16), the second condition of (42) can be conveniently written as

$$
1+s\left(1-F_{d}^{-1}\right)^{-s}=0 .
$$

This gives $s=s\left(F_{d}\right)$ as a function of Froude number. In the cases when this equation does not have a real solution, we determine the control parameter $s$ (which, we recall, is more general entity than the critical index) from the minimization of $\Psi_{2}^{*}\left(t_{c}, s\right)$ :

$$
\min _{s}\left(1+s\left(1-F_{d}^{-1}\right)^{-s}\right)
$$

The Yukalov-Gluzman technique then confronts the two approximants $\Psi_{1}^{*}$ and $\Psi_{2}^{*}$ : after their difference is minimized, it remains to decide which one of them is the best re-summed expression originating from the original perturbative expansion. Implicit in this approach is the concept that the renormalization approach might not be fully convergent asymptotically but only locally. Such a decision can be made based on the analysis of the corresponding multipliers

$$
M_{j}(t, s) \equiv\left[\frac{\partial \Psi_{j}^{*}(\varphi, s)}{\partial \varphi}\right]_{\varphi=P_{1}(t, 0)}, \quad j=1,2
$$

yielding

$$
\begin{gathered}
M_{1}(t, s)=\left(1-\frac{a_{1} t}{s}\right)^{-(1+s)}, \\
M_{2}(t, s)=\left(1-\frac{a_{2}}{a_{1} s} t\right)^{-s}\left[1+\frac{a_{2}}{a_{1}} t\left(1-\frac{a_{2}}{a_{1} s} t\right)^{-1}\right] .
\end{gathered}
$$

The more stable solution corresponding to the smallest $\left|M_{j}\right|$ should then be selected. We find in general that $\Psi_{1}^{*}$ has the smallest multiplier in the critical region, which onset is determined by the condition $\left|M_{1}(t, s)\right|<<\left|M_{2}(t, s)\right|$, provided that a solution to (44) or (45) exists. On the other hand, we find that $\Psi_{2}^{*}$ prevails in some "pseudocritical" regime when the first solution $\Psi_{1}^{*}$ becomes unstable. One can make this selection process automatic by the weighting procedure advocated in [30] which has also been used in [32]. The weighting procedure amounts to defining an average of the two approximants with weights inversely proportional to their multipliers. The rational for this approach is that the inverse of the multipliers can be shown to play a role similar to the probability that the system visits the dynamical state described by the corresponding approximant. The resulting function is

$$
\Psi^{*}(t, s)=\frac{\Psi_{1}^{*}(t, s)\left|M_{1}(t, s)\right|^{-1}+\Psi_{2}^{*}(t, s)\left|M_{2}(t, s)\right|^{-1}}{\left|M_{1}(t, s)\right|^{-1}+\left|M_{2}(t, s)\right|^{-1}} .
$$


Since we are interested in the physically meaningful case when the critical time $t_{c}=s / a_{1}$ is positive (it may be infinite), a modification is required when $s / a_{1}$ is found negative or simply where there are no real solution to (42). We take this situation as a signal that one should use the inverse function defined by (18) as the relevant expansion to obtain the most stable scenario. The sought function is then defined as the inverse of the weighted-average for inverse renormalized approximant. The approximants and corresponding multipliers are calculated using the parameters $a_{i}$ 's (equation (15)) changed into $b_{i}$ 's (equation (18)). The final solution reads

$$
\Psi^{*}(t, s) \equiv \Psi_{I}^{*}(t, s)^{-1}=\left(\frac{\Psi_{1 I}^{*}(t, s)\left|M_{1 I}(t, s)\right|^{-1}+\Psi_{2 I}^{*}(t, s)\left|M_{2 I}(t, s)\right|^{-1}}{\left|M_{1 I}(t, s)\right|^{-1}+\left|M_{2 I}(t, s)\right|^{-1}}\right)^{-1} .
$$

\subsection{Negative velocity $a_{1}<0$ (downward trend)}

Using the general procedure of section 6.1, we now present the corresponding classification of the different possible regimes.

\subsubsection{Negative acceleration $a_{2}<0$}

In this situation, the inverse function has always a singularity. This corresponds for the direct observable function to vanish in finite time (critical regime I) at $t_{c}=s\left(F_{d}\right) / a_{1}$, where $s\left(F_{d}\right)$ is the negative solution of equation (44). Both approximants $\Psi_{1}^{*}$ and $\Psi_{2}^{*}$ contribute to the expression (49) and $\Psi_{2}^{*}$ progressively dominates at time approaches $t_{c}$.

Solution $\Psi_{2}^{*}$ contribute to the average (48) more than $\Psi_{1}^{*}$, because $\left|M_{2}\right|$ always smaller than $\left|M_{1}\right|$. As the multiplier of $\Psi_{1}^{*}(t)$ eventually blows up to infinity at $t_{c}, \Psi_{2}^{*}(t)$ ends by dominating the behavior of $\Psi^{*}(t)$.

The asymptotic behavior of the average close to $t_{c}$ is determined by $\Psi_{2}^{*}$ and is characterized by exponent $z=$ 1 , notwithstanding the fact that the control exponent $s$ is fractional. This corresponds to the situation where the observable goes to zero linearly in time.

\subsubsection{Positive moderate acceleration $0<a_{2}<\frac{a_{1}^{2}}{F_{0}}, F_{0}<F_{d}$ where $F_{0}=\left(1-e^{-1 / e}\right)^{-1}$}

In this region of parameter $F_{0}=\left(1-e^{-1 / e}\right)^{-1}=3.249<F_{d}$, the observable still goes to zero in finite time (critical regime I). The time $t_{c}$ at which the observable vanishes is given by the same formula as in section 6.2.1. This solution exists as long as there is solution $s\left(F_{d}\right)$ to (44). When $F_{d}$ becomes too small, (44) has no more any solution and this corresponds to the critical regime II discussed in the next section. The boundary between these two regimes occurs at the Froude value $F_{0}$ determined by adding the condition

$$
\frac{\partial\left(\Psi_{2}^{*}\left(s / a_{1}, s\right)\right)}{\partial s}=0
$$

to the general equation $\Psi_{2}^{*}\left(s / a_{1}, s\right)=0$. The minimum, solution of (50), is located at

$$
s_{\min }=\frac{1}{\ln \left(\frac{F_{d}-1}{F_{d}}\right)},
$$

and coincides with the zero of $\Psi_{2}^{*}$ only for the specific value of the Froude number $F_{0}$ thus determined by

$$
F_{0}=\left(1-e^{-1 / e}\right)^{-1}=3.249 .
$$

As $F_{d} \rightarrow \infty$, equation (44) can be solved exactly and $s\left(F_{d} \rightarrow \infty\right)=-1$, which is a mean-field value $z=1$. In other words, given in advance a linear function $\phi(t)=1-\left|a_{1}\right| t$, our technique will indeed reconstruct it! Let us expand Eq.(35) around this exactly solvable limit in powers of a small parameter $1 / F_{d}=y$,

$$
1+s\left(1-F_{d}^{-1}\right)^{-s} \simeq 1+s+s^{2} y+\ldots
$$


Then,

$$
s=\frac{1}{2 y}(-1+\sqrt{1-4 y}) \simeq-1-y+\ldots(y \rightarrow 0) .
$$

This expression breaks down around $F_{d}=4$, where the whole equation (44) should be considered down to $F_{0}$, where $s\left(F_{0}\right)=-e$.

Solution $\Psi_{1}^{*}$ starts to contribute to the average (48) more than $\Psi_{2}^{*}$, as soon as $t$ satisfy condition $\left|M_{1}\right| \ll\left|M_{2}\right|$, as shown in Fig. 1, which represents the dependence of the two approximants $\Psi_{1}^{*}(t)$ (equation (40)) and $\Psi_{2}^{*}(t)$ (equation (41)) and of their weighted average $\Psi^{*}(t)$ given by (48). As the multiplier of $\Psi_{1}^{*}(t)$ eventually vanishes at $t_{c}, \Psi_{1}^{*}(t)$ ends by dominating the behavior of $\Psi^{*}(t)$ and the average demonstrates critical behavior with positive fractional exponent $z=-s\left(F_{d}\right)$. Thus, in this region of $F_{d}$, as $t$ goes to $t_{c}$, we obtain a critical behavior with fractional $s$ playing the role of critical index $z$. It means that the exponent is now different from -1 and is determined by the solution of eq. (44).

\subsubsection{Strong positive acceleration $\frac{a_{1}^{2}}{F_{0}}<a_{2}, F_{d}<F_{0}$}

The critical regime (I) is now replaced by the critical regime (II), such that equation (44) has no more any solution and the control exponent and critical time are determined from the minimization of eq. (45), which gives

$$
s=s_{\min }\left(F_{d}\right)=\frac{1}{\ln \left(\frac{F_{d}-1}{F_{d}}\right)} .
$$

The critical index is $z=-s_{\min }\left(F_{d}\right)$, leading to a logarithmic correction to the mean-field value. The critical time is given by

$$
t_{c}=\frac{s_{\min }\left(F_{d}\right)}{a_{1}} .
$$

Figure 2 shows the dependence of the two approximants $\Psi_{1}^{*}(t)$ (equation (40)) and $\Psi_{2}^{*}(t)$ (equation (41)) and of their weighted average $\Psi^{*}(t)$ given by (48). The new feature is the existence of a minimum at time $t_{\min }$ of $\Psi_{2}^{*}(t)$ and, therefore of a non-monotonous behavior also of the average $\Psi^{*}(t)$, with $t_{\min }$ given by

$$
t_{\min }=-\frac{a_{1}}{a_{2}} \frac{s_{\min }}{s_{\min }-1},
$$

corrected by the ratio $\frac{s_{\min }}{s_{\min }-1}$ compared to the mean-field result (23) of section 3.2 .3 . The value of $\Psi_{2}^{*}(t)$ at $t_{\min }$ is

$$
\Psi_{2 \min }^{*}=1-F_{d}\left(1+L\left(F_{d}\right)\right)^{-\left(1+L\left(F_{d}\right)\right) / L\left(F_{d}\right)}, \quad L\left(F_{d}\right)=\ln \left(\frac{F_{d}}{F_{d}-1}\right) .
$$

The trajectory $\Psi^{*}(t)$ shown in figure 2 is rather unusual since after spending some time close to the local minimum of a non-critical branch $\Psi_{2}^{*}(t)$, the system suddenly breaks down towards the "critical" branch $\Psi_{1}^{*}(t)$, which then ends at a critical point $t_{c}$. This means that the critical behavior with exponent $z=-s_{\min }\left(F_{d}\right)$ has not disappeared yet. The drop occurs at a crossover time $t=t_{\text {cros }}$ defined as the solution to the equation $\left|M_{1}\right| \approx\left|M_{2}\right|$, with a magnitude $\Delta=\Psi_{2}^{*}\left(t_{\text {cros }}\right)-\Psi_{1}^{*}\left(t_{\text {cros }}\right)$. This regime is found for the Froude interval $F_{01}<F_{d}<F_{0}$ where $F_{01}=\left(1-e^{-1}\right)^{-1}=1.582$ is the solution of the equation

$$
1+\frac{1}{\ln \left(\frac{F_{01}-1}{F_{01}}\right)}=0
$$

corresponding to the Froude value at which the multiplier $M_{1}(t, s)$ changes from stable $\left(M_{1}<1\right)$ to unstable $\left(M_{1}>\right.$ 1) behavior. As the regime $1<F_{d}<F_{01}$ (pseudocritical regime I) sets in, the multiplier $M_{1}(t, s)$ becomes larger than 1 , increases with time and diverges at $t_{c}$. Rather than converging to the $\Psi_{1}^{*}(t)$ approximant, the weighted average 
$\Psi^{*}(t)$ exhibits a fast change of direction to reach $\Psi_{2}^{*}(t)$ at $t_{c}$, as shown in figure 3 . The critical branch has disappeared as the non-critical branch $\Psi_{2}^{*}$ dominates. The presence of the approximant scenario $\Psi_{1}^{*}$ is felt only in the existence of some oscillations decorating the $\Psi_{2}^{*}$ scenario. This regime exists for $1<F_{d}<F_{01}$.

For $F_{d}<1$ ( pseudocritical regime II), the minimum of (45) disappears and there is no solution either to (44) or to (45) anymore. This implies that we should use the inverse expansion in terms of the coefficients $b_{1}>0, b_{2}<$ $0\left(F_{I}<0\right)$. The corresponding control exponent $s$ is then obtained from the condition

$$
1+s\left(1-F_{I}^{-1}\right)^{-s}=0,
$$

which has a negative solution $s=s\left(F_{I}\right)$ leading to $t_{c}=s / b_{1}<0$, which is not allowed. However, similarly to the previous strategy to replace (44) by (45), we can look for the solution which minimizes the left-hand-side of (59), which gives $s \rightarrow \infty$. This corresponds to a pseudocritical regime which is reminiscent of the last phase of the previous regime, but with formally infinite $t_{c}$. In the limit $s \rightarrow \infty$, we obtain

$$
\begin{gathered}
\Psi_{1 I}^{*}(t)=\exp \left(b_{1} t\right), \quad M_{1 I}(t)=\exp \left(b_{1} t\right) \\
\Psi_{2 I}^{*}(t)=1+b_{1} t \exp \left(\frac{b_{2}}{b_{1}} t\right), \quad M_{2 I}(t)=\left(1+\frac{b_{2}}{b_{1}} t\right) \exp \left(\frac{b_{2}}{b_{1}} t\right) .
\end{gathered}
$$

Note that $\Psi_{2 I}^{*}(t)$ is qualitatively similar to the mean-field solution of section III, derived for the same region of parameters. The multiplier $M_{1 I}(t)$ is always larger than 1 which implies that the scenario $\Psi_{2 I}^{*}$ always dominates in the weighted average, although the contribution of $\Psi_{1 I}^{*}$ is responsible for the existence of an extra- minimum in the trajectory of $\Psi^{*}$ given by expression (49), as shown in figure 4.

\subsection{Positive velocity $a_{1}>0$ (upward trend)}

\subsubsection{Negative acceleration: $a_{2}<0$}

One can still define the control exponent $s$ from the condition (44), but $t_{c}$ becomes negative which is undesirable. As in the previous section, we turn to the next possibility which is to use (45), whose only solution is $s \rightarrow+\infty$. This solution turns out to minimize the difference between two approximant scenarios. This regime is the inversion of the pseudocritical regime II just presented above (only with $F_{d}<0$ instead of $F_{I}$ ), as it is described by the following solutions,

$$
\begin{gathered}
\Psi_{1}^{*}(t)=\exp \left(a_{1} t\right), \quad M_{1}(t)=\exp \left(a_{1} t\right) . \\
\Psi_{2}^{*}(t)=1+a_{1} t \exp \left(\frac{a_{2}}{a_{1}} t\right), \quad M_{2}(t)=\left(1+\frac{a_{2}}{a_{1}} t\right) \exp \left(\frac{a_{2}}{a_{1}} t\right) .
\end{gathered}
$$

Note that $\Psi_{2}^{*}(t)$ is nothing but the mean-field solution of section III, derived for the same parameter region. The multiplier $M_{1}(t)$ is found to be always larger than 1 . Therefore, $\Psi_{2}^{*}$ dominates in the weighted average trajectory $\Psi^{*}(48)$. The contribution from $\Psi_{1}^{*}$ induces splitting of the mean-field maximum (at $t=-a_{1} / a_{2}$ ) as shown in figure 5.

\subsubsection{Moderate positive acceleration: $0<a_{2}<\frac{F_{0}-1}{F_{0}} a_{1}^{2} ; \frac{F_{0}}{F_{0}-1}<F_{d}$}

In this case, although equation (44) has a solution for $s<0$, the corresponding $t_{c}$ is negative. After inversion of the initial series, this region of parameters is equivalent to $1<F_{I}<F_{0}$. This regime corresponds to the inverse of the critical regime II described above and can be described similarly.

Consider first the region of $F_{01}<F_{I}<F_{0}$. There is a minimum of the curve $\Psi_{2 I}^{*}$ and the shape of the observable $\left(\Psi_{I}^{*}\right)^{-1}$ (see equation (49) ) is significantly non-monotonous, due to contribution from $\Psi_{2 I}^{*}$. The time $t_{\min }$ of the minimum of $\left(\Psi_{2 I}^{*}\right)^{-1}$ (maximum of $\Psi_{2 I}^{*}$ ) is given by

$$
t_{\min }=-\frac{b_{1}}{b_{2}} \frac{s_{\min }}{s_{\min }-1},
$$


where

$$
s_{\min }=\frac{1}{\ln \left(\frac{F_{I}-1}{F_{I}}\right)}
$$

is the solution of the minimization

$$
\min _{s}\left(1+s\left(1-F_{I}^{-1}\right)^{-s}\right)
$$

The maximum value is $\left(\Psi_{2 I(\min )}^{*}\right)^{-1}$ where

$$
\Psi_{2 I(\min )}^{*}=\left(1-F_{I}\left(1+L\left(F_{I}\right)\right)^{-\left(1+L\left(F_{I}\right)\right) / L\left(F_{I}\right)}\right), \quad L\left(F_{I}\right)=\ln \left(\frac{F_{I}}{F_{I}-1}\right) .
$$

The trajectory has the same topology as shown in figure 2 except that it is the inverse of the function shown in figure 2. The observable is predicted as the weighted average scenario given by (49) and is shown in figure 6 . The "critical" branch $\Psi_{1 I}^{*}$ shapes the weighted average $\Psi_{I}^{*}$ as $t_{c}$ is approached. The weighted average scenario goes to infinity in finite time $t_{c}=s_{\min } / b_{1}$, with negative $z=s_{\min }$ describing the power-low divergence. In terms of the coefficients $a_{i}$ of the polynomial expansion, this regime holds for $\frac{F_{01}-1}{F_{01}} a_{1}^{2}<a_{2}<\frac{F_{0}-1}{F_{0}} a_{1}^{2}$, i.e., for $\frac{F_{0}}{F_{0}-1}<F_{d}<\frac{F_{01}}{F_{01}-1}$ $\left(\frac{F_{01}-1}{F_{01}}=0.368, \frac{F_{0}-1}{F_{0}}=0.692\right)$. These conditions are equivalent to $F_{01}<F_{I}<F_{0}$.

As $F_{I}$ becomes smaller than $F_{01}$, the multiplier $M_{1 I}(\mathrm{t}, \mathrm{s})$ changes from stable $\left(M_{1 I}<1\right)$ to unstable $\left(M_{1 I}>1\right)$. As a consequence and similarly to the change from figure 2 to figure 3, the weighted average scenario changes dramatically and does not exhibit anymore a critical divergence at $t_{c}$. The scenario $\Psi_{1 I}^{*}$ is felt only in the creation of a few oscillations around $\Psi_{2 I}^{*}$. This regime holds for $1<F_{I}<F_{01}$ and mirrors the pseudocritical regime I previously described. In terms of initial coefficients, it corresponds to $0<a_{2}<\frac{F_{01}-1}{F_{01}} a_{1}^{2}$.

6.3.3 Large positive acceleration: $\frac{F_{0}-1}{F_{0}} a_{1}^{2}<a_{2}, F_{I}>F_{0}$

In terms of the inverse Froude number, this regime holds for $F_{I}>F_{0}$. The observable goes to infinity in finite time at a critical time $t_{c}$, which is determined from the condition that the inverse quantities cross zero. The corresponding control exponent $s\left(F_{I}\right)$ is the solution of

$$
1+s\left(1-F_{I}^{-1}\right)^{-s}=0
$$

and

$$
t_{c}=s\left(F_{I}\right) / b_{1} .
$$

The existence of the finite-time singularity holds as long as $F_{I}>F_{0}$. This condition can be re-expressed in terms of the direct Froude number and gives $F_{d}<\frac{F_{0}}{F_{0}-1}=1.445$. This regime mirrors the critical regime I. Given a secondorder expansion $\phi_{2}(t) \simeq 1+\left|a_{1}\right| t+\left|a_{1}\right|^{2} t^{2}$ of a simple pole $\phi(t)=\left(1-\left|a_{1}\right| t\right)^{-1}$ (with $F_{I} \rightarrow \infty$ ), our technique will indeed reconstruct it! The weighted average scenario $\Psi_{I}^{*}(t, s)^{-1}$ goes to infinity in finite time $t_{c}$, with negative $z=s\left(F_{I}\right)$ describing the power-low divergence. In Fig. 7 we demonstrate the dependence of the two approximants $\Psi_{1}^{*}(t)$ and $\Psi_{2}^{*}(t)$ and of their weighted average $\Psi^{*}(t)$ given by (49) .

\section{Concluding Remark}

Starting from a representation of the early time evolution of a dynamical system in terms of the polynomial expression of some observable $\phi(t)$ as a function of time, we have investigated the conditions under which this early time dynamics may lead or may not lead to a finite-time singularity. The corresponding classification has been performed from the point of view of the functional renormalization method of Yukalov and Gluzman [18]-[30], with the purpose of identifying the most stable scenarios, given the early time dynamics. The direct extension of this work is to test our predictions empirically, following the methodology of [16] developed for a particular case. 


\section{References}

[1] A. Pumir, E. D. Siggia, Phys. Rev. A 45, R5351 (1992).

[2] A. Bhattacharjee, A., C.S. Ng and X. Phys. Rev. E 52, 5110 (1995).

[3] N. M. Zubarev, Phys. Lett. A 243, 128 (1998).

[4] X. Leoncini, L. Kuznetsov, G. M. Zaslavsky Fluids 12, 1911 (2000).

[5] M. W. Choptuik, Phys. Rev. Lett. 70, 9 (2000); Progress of Theoretical Physics Supplement 136, 353 (1999).

[6] M. Rascle, C. Ziti, Journal of Mathematical Biology 33, 388 (1995).

[7] H. K. Moffatt, Nature 404, 833 (2000).

[8] A.A Stanislavsky, and K. Weron, Physica D 156, 247 (2001).

[9] A. Johansen, and D. Sornette, Int. J. Mod. Phys. C 9, 433 (1998).

[10] A. Johansen, and D. Sornette Eur. Phys. J. B 18, 163 (2000)

[11] R. C. Hidalgo, F. Kun, and H.J. Herrmann, preprint at cond-mat/0103232 (2001).

[12] A. Johansen, Saleur, H., Sornette, D. Eur. Phys. J. B 15, 551 and references therein (2000).

[13] S. G. Sammis, and D. Sornette, Proceedings of the National Academy of Sciences USA, in press (2001) (http://arXiv.org/abs/cond-mat/0107143)

[14] A. Johansen, Sornette, D. Ledoit, O, Journal of Risk 1, 5 and references therein (1999).

[15] A. Johansen A. and Sornette D, Eur. Phys. J. B 17, 319 (2000).

[16] S. Gluzman, J.V. Andersen and D. Sornette, Computational Seismology 32, 122 (2001).

[17] C. Ziehmann, L.A. Smith and J. Kurths, Phys. Letts. 271, 237 (2000).

[18] V. I. Yukalov, Phys. Rev. A 42, 3324 (1990).

[19] V. I. Yukalov, J. Math. Phys. 32, 1235 (1991).

[20] V. I. Yukalov, J. Math. Phys. 33, 3994 (1992).

[21] V. I. Yukalov and Yukalova E.P, Physica A 206, 553 (1994).

[22] V. I. Yukalov, and Yukalova E.P, Physica A 225, 336 (1996).

[23] S. Gluzman, and V.I Yukalov (1997), Phys. Rev. E 55, 3983 (1997).

[24] V. I. Yukalov, and S. Gluzman, Phys. Rev. E 55, 6552 (1997).

[25] V. I. Yukalov, and S. Gluzman, Phys. Rev. Lett. 79, 333 (1997).

[26] V. I. Yukalov, and S. Gluzman, Phys. Rev. E 58, 1359 (1998).

[27] S. Gluzman and V. I. Yukalov, Phys. Rev. E 58, 4197 (1998).

[28] V. I. Yukalov, and S. Gluzman, Physica A 273, 401 (1999). 
[29] V. I. Yukalov, E.P. Yukalova and S. Gluzman, Phys. Rev. A 58, 96 (1998).

[30] V. I. Yukalov and S. Gluzman, Int. J. of Mod. Phys. B 13, 1463 (1999).

[31] L. V. Kantorovich, and G.P. Akilov, Functional analysis in normed spaces (A Pergamon Press Book, The MacMillan Company, New York, 1964).

[32] J. V. Andersen, S. Gluzman and D. Sornette, Eur. Phys. J. B 14, 579 (2000).

[33] S. Gluzman, and D. Sornette, Phys. Rev. E V6306 N6 PT2:6129,U241 (2001). 


\section{Figures captions}

Fig. 1: First-order approximant $\Psi_{1}^{*}$ (equation (40)), dashed line), second-order approximant $\Psi_{2}^{*}$ (equation (41), dotted line) and their weighted average given by (48) (continuous line) as a function of time, for positive moderate acceleration $0<a_{2}<\frac{a_{1}^{2}}{F_{0}}, F_{0}<F_{d}$ where $F_{0}=\left(1-e^{-1 / e}\right)^{-1}$.

Fig. 2: Dependence of the two approximants $\Psi_{1}^{*}(t)$ (equation (40), dashed line) and $\Psi_{2}^{*}(t)$ (equation (41), dotted line) and of their weighted average $\Psi^{*}(t)$ given by (48) (continuous line) in the regime of strong positive acceleration $\frac{a_{1}^{2}}{F_{0}}<a_{2}, F_{d}<F_{0}$.

Fig. 3: Same as figure 2 in the regime $1<F_{d}<F_{01}$ (pseudocritical regime I). Note that the weighted average $\Psi^{*}(t)$ exhibits a fast change of direction to reach $\Psi_{2}^{*}(t)$ at $t_{c}$.

Fig. 4: Same as figure 2 for $F_{d}<1$.

Fig. 5: Same as figure 2 for a negative acceleration $a_{2}<0$.

Fig. 6: Same as figure 2 in the regime of moderate positive acceleration: $0<a_{2}<\frac{F_{0}-1}{F_{0}} a_{1}^{2} ; \frac{F_{0}}{F_{0}-1}<F_{d}$.

Fig. 7: Same as figure 2 in the regime $\frac{F_{0}-1}{F_{0}} a_{1}^{2}<a_{2}, F_{I}>F_{0}$. 
Fig. 1

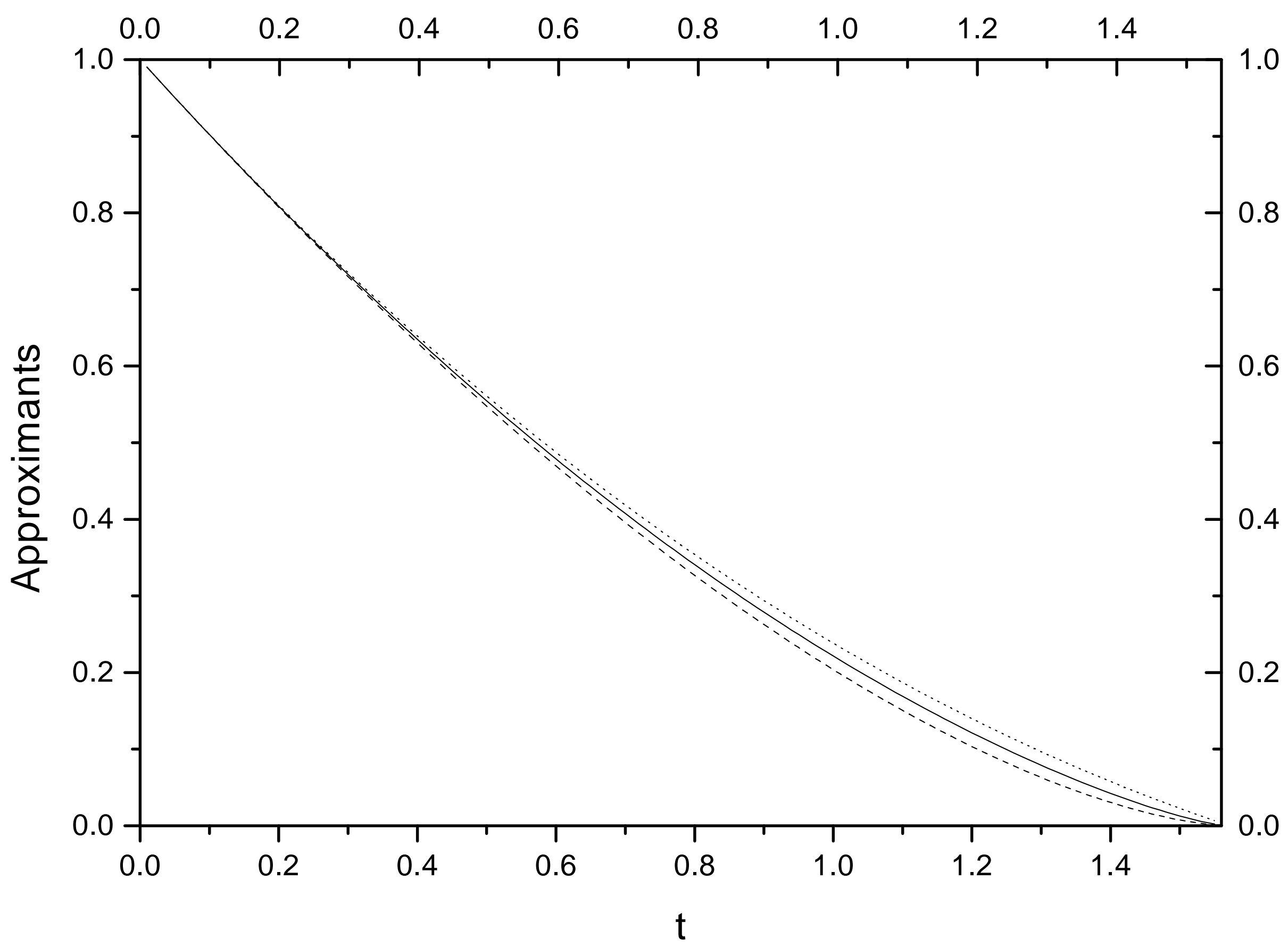


Fig. 2

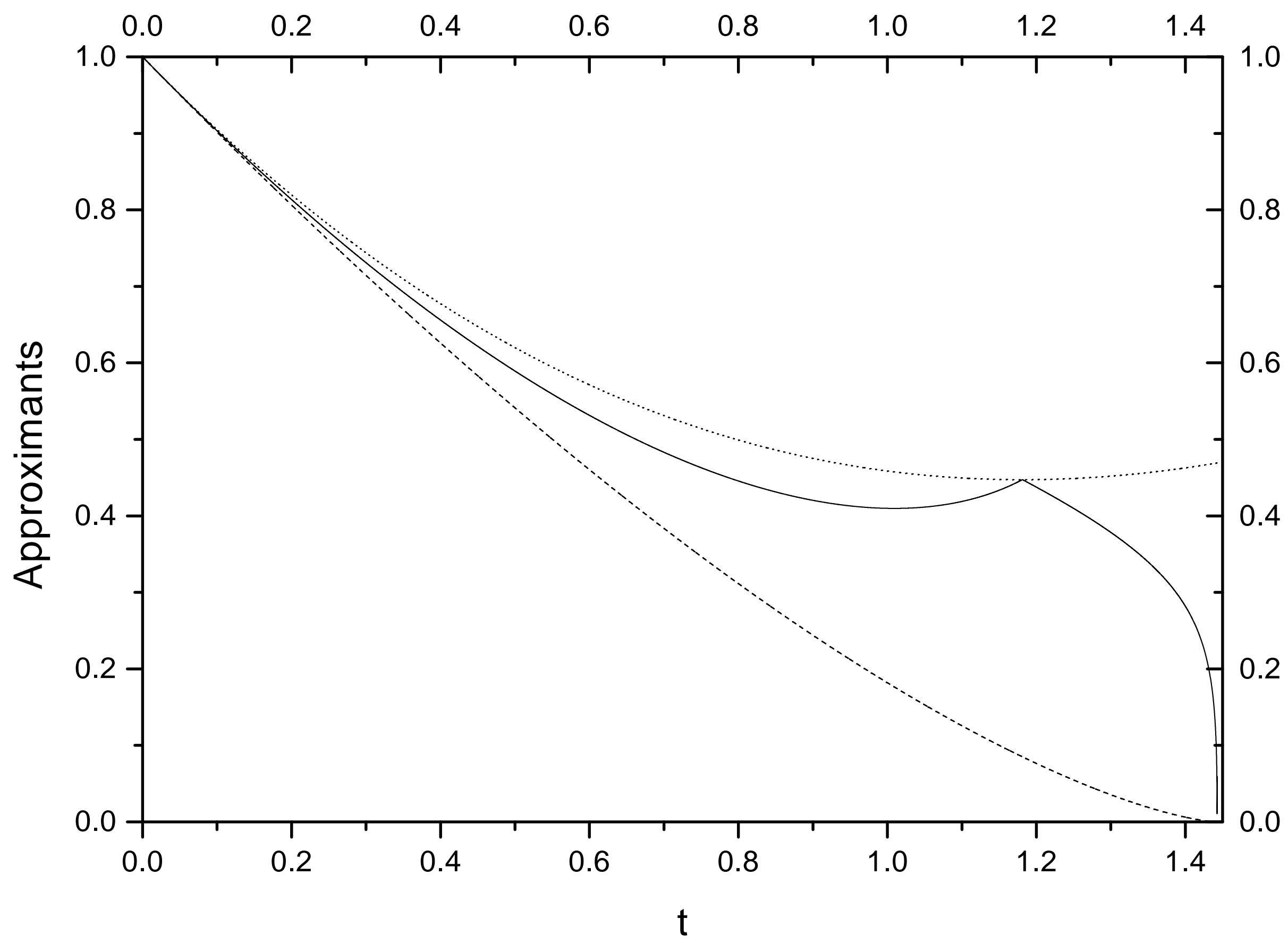


Fig. 3

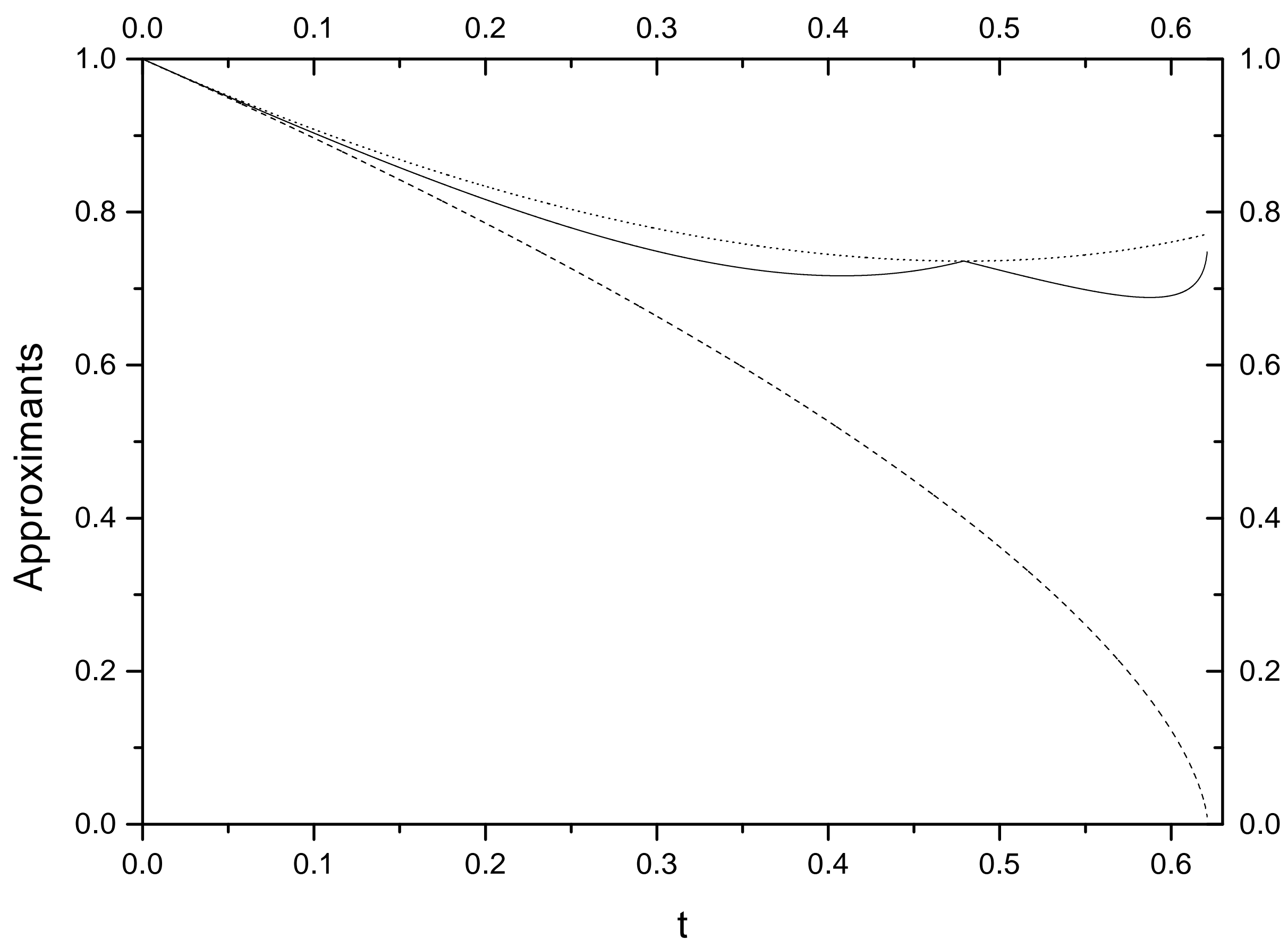


Fig. 4

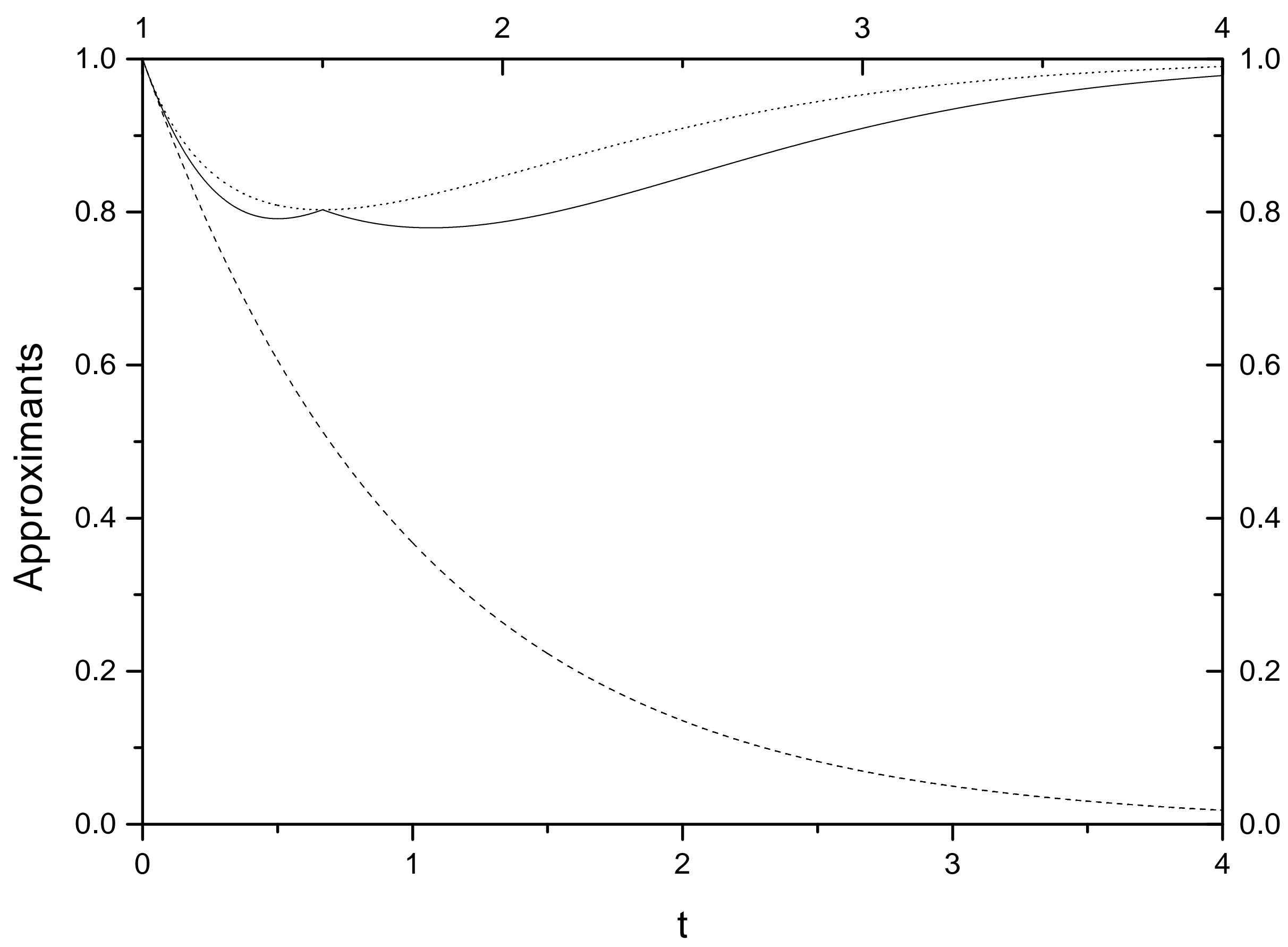


Fig. 5

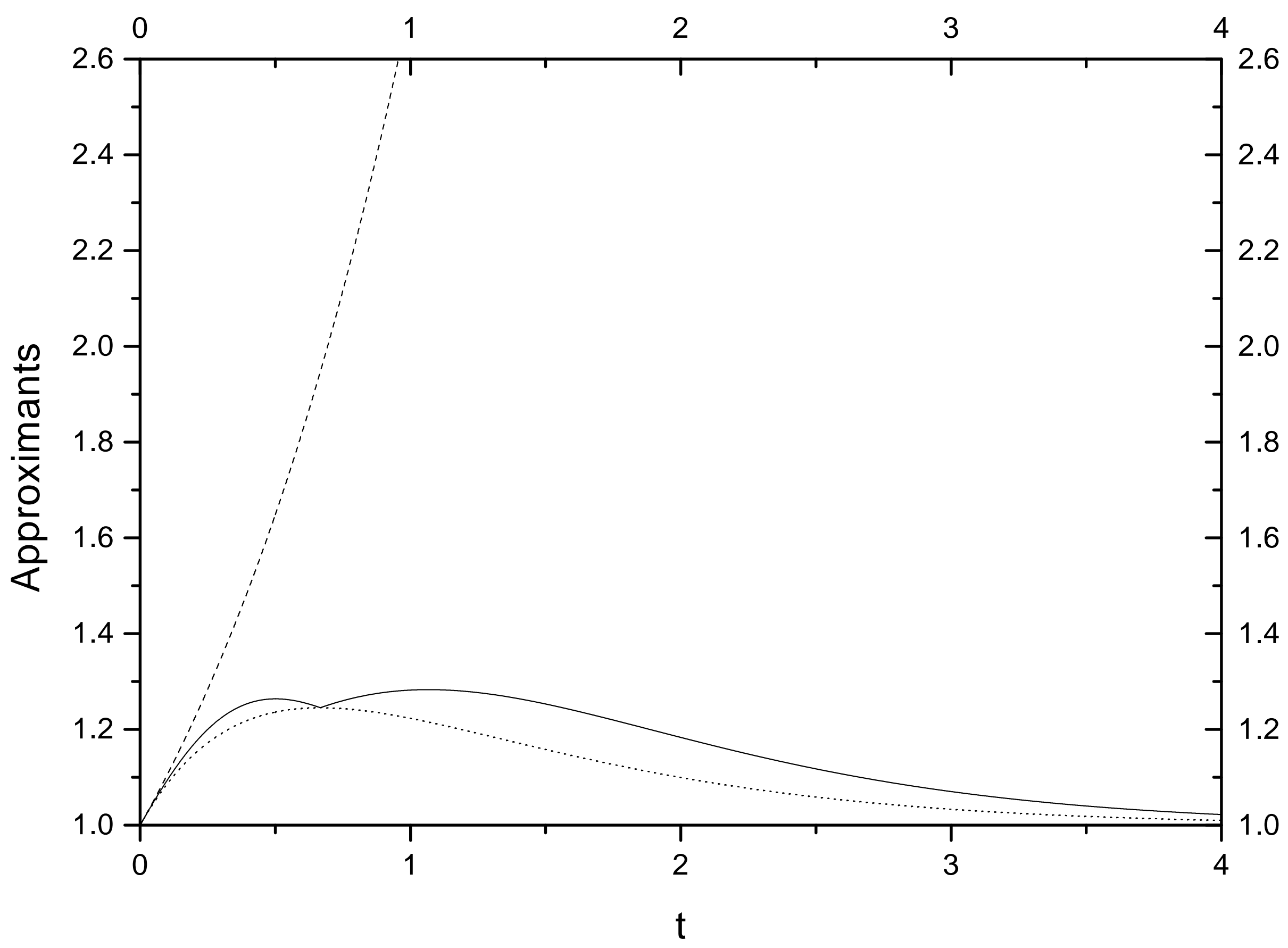


Fig. 6

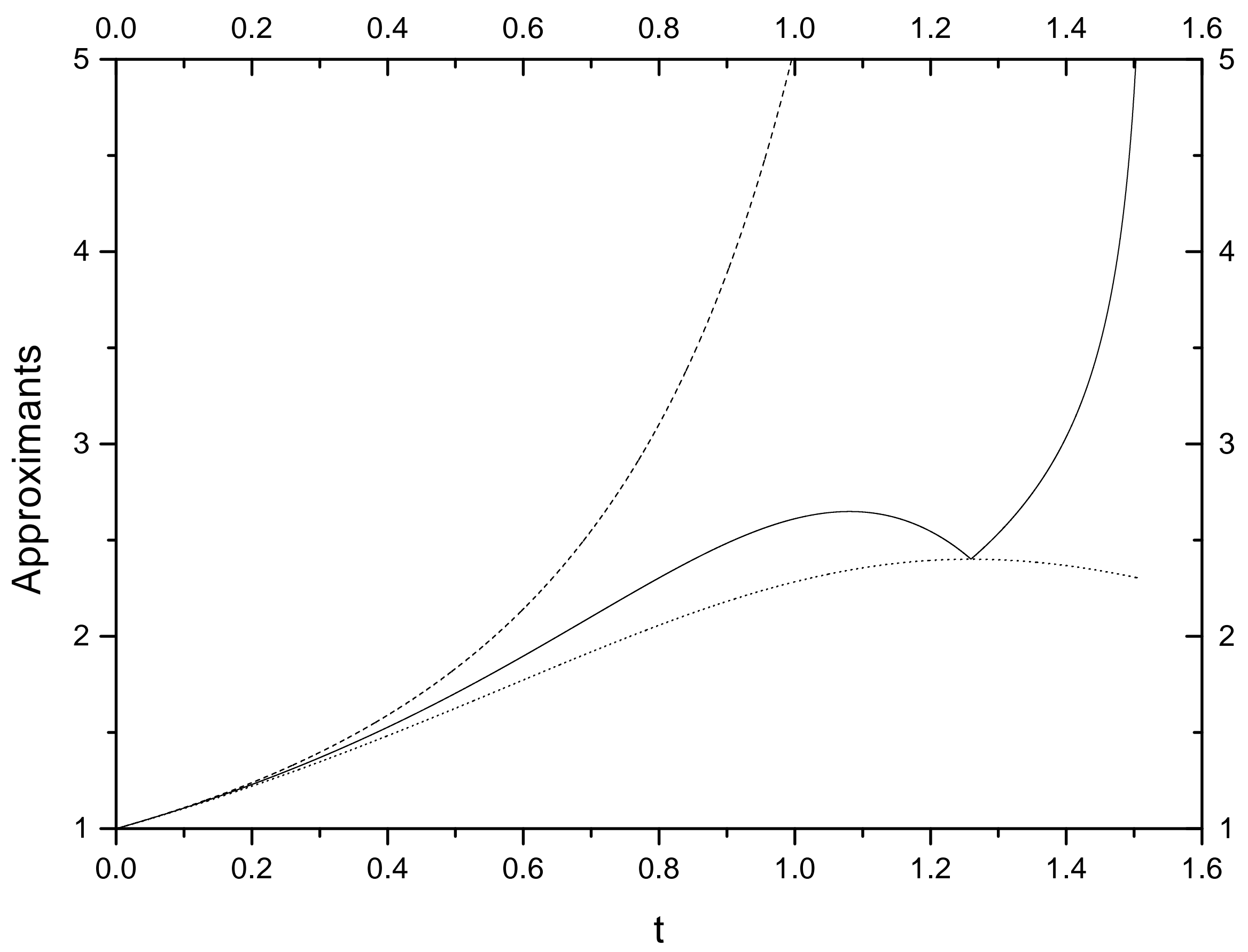


Fig. 7

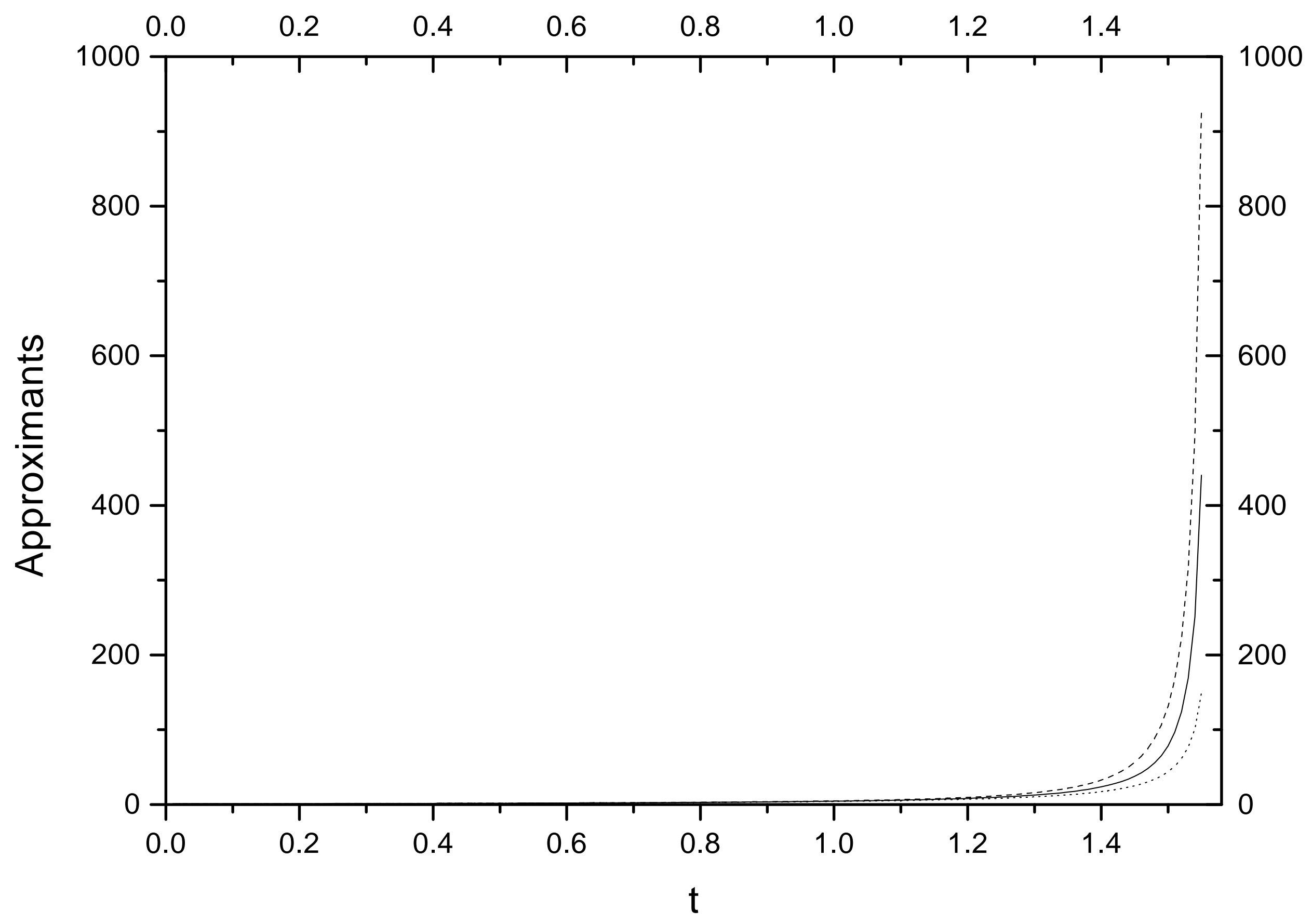

